\title{
SUGARCANE PRODUCTIVITY ESTIMATION THROUGH PROCESSING HYPERSPECTRAL SIGNATURES USING ARTIFICIAL NEURAL NETWORKS
}

\author{
C.E. Espinosa ${ }^{1}$, S. Velásquez ${ }^{1}$, F. L. Hernández ${ }^{1 *}$ \\ ${ }^{1}$ Remote Sensing Research Group, Universidad del Valle, Santiago de Cali, Colombia \\ (espinosa.camilo, sergio.velasquez, francisco.hernandez)@correounivalle.edu.co
}

KEY WORDS: Neural Networks, Net Primary Productivity, Deep Learning, Backpropagation, Hyperspectral Signatures, Sugarcane.

\begin{abstract}
:
This project uses an artificial neural network to calculate the net primary productivity of an organic sugarcane crop in Hatico's farm, in Cerrito, Valle del Cauca. The pilot scheme used in this project is composed by 6 treatments of nitrogen fertilization based on green manures (poultry manure and cowpea). During the last two crops' phenological phases, the artificial neural network was provided with hyperspectral data collected in the field. In addition, an exploratory data study was implemented in order to identify anomalous signs related to the light saturation and the curvature geometry. The first network applied was Autoencoder, in order to reduce the dimensionality of the radiometric resolution of the data. The second network applied was Multilayer Perceptron (MLP), to calculate the productivity values of the patches. After having compared the actual productivity values provided by Cenicaña, this project obtained an accuracy of $91.23 \%$ in the productivity predictions.
\end{abstract}

\section{INTRODUCTION}

Sugarcane is one of the perennial crops with the highest organic matter rate per unit area, as a result, it is one of the most productive crops in the agricultural market around the world (Duveiller et al., 2013). Colombia is placed 13th among the sugarcane-producing countries and has the first position in productivity. The Colombian sugar sector is located in the valley of the Cauca's river, which covers 47 municipalities from the north of Cauca and central Valle del Cauca, to the south of Risaralda. In this region there are 225,560 hectares planted with sugarcane; $25 \%$ of them belong to sugar mills and the remaining $75 \%$ correspond to around 2,750 cane growers (Asocaña, 2018).

Biomass estimation methods can be categorized as either destructive or non-destructive sampling techniques. Remote sensing is a non-destructive sampling defined as a set of techniques used to read an object's spectral information based on the way it interacts with energy, which is recorded by sensors (Espín, 2015).

Remote sensing has facilitated crops supervision by providing permanent condition data in large areas (Bégué, et al., 2010). However, the multispectral data obtained by satellite or drone imagery can't be as detailed as the hyperspectral data (Abdel-Rahman, et al., 2009). Spectrometry techniques allow to obtain hyperspectral data along the electromagnetic spectrum, showing significant spectral patterns in different regions, which are related to plant phenology and are used to facilitate both management and productivity estimation (Curran, Dungan \& Peterson, 2001).

Artificial neural networks (ANN) are an automatic learning model based on biological neural networks and connections. These systems consist of a set of elements or neurons that connect to each other to send information to each of the nodes where the error spreads depending on the weight of the connections. There are different designs of neural networks, which can be used to perform many activities. The multilayer perceptron (MLP) model is frequently used in Deep Learning, due to its potential to classify, to predict, and to how easy it is to operate (Caicedo \& López, 2009).

MLP is based on a backpropagation algorithm, which distributes the error of the output layer, in the hidden layers. This network architecture is made up of an input layer, at least a hidden and an output layer. The connections between these types of neural networks are usually either FeedForWard or sequential type, which means that all the neurons in the input layer are connected to every neuron in the hidden layer. On the other hand, autoencoders are a type of neural network that uses the same sequential model used by the MLP, however, its design varies by conserving a smaller number of neurons in the hidden layers than in the output layers. Autoencoders are based on the dimensionality reduction obtained from principal component analysis (PCA).

PCA take the most important features in the input data and reduce them through linear transformations. Although the autoencoders and the PCA are similar, the autoencoders employ a nonlinear component analysis, due to the high variety of data. The Autoencoder is composed of two regions, encoding, where the data is transformed reducing its dimensionality and then transporting the essential information from the data group to the hidden layers (NG, 2011). The decoding layer is the network part where the data is reconstructed following the input layer scheme. 
This study applies the net primary productivity model (PPN) proposed by Kumar \& Monteith, (1981), which is based on the plant's response to photo synthetically active radiation (PAR) and the most determining factors of the crop, such as environmental variables, plant phenology and physiology and behavior throughout time. The values of PPN, the set of hyperspectral data and the information of the physiological variables of the crop, were used to provide a neural network that allowed to estimate the productivity.

\section{STUDY AREA AND MATERIALS}

\subsection{Study Area}

The study area is identified as the 758B crop, located at Hatico's farm, in Cerrito, Valle del Cauca, Colombia. The study area is located in the geographical coordinates $3^{\circ} 38^{\prime} 24^{\prime \prime} \mathrm{N}, 76^{\circ} 19^{\prime} 48^{\prime \prime} \mathrm{W}$. The average temperature of the region is approximately $25^{\circ} \mathrm{C}$. The $758 \mathrm{~B}$ crop corresponds to an experimental sugarcane crop that contained 30 organic patches as presented in Figure 1.

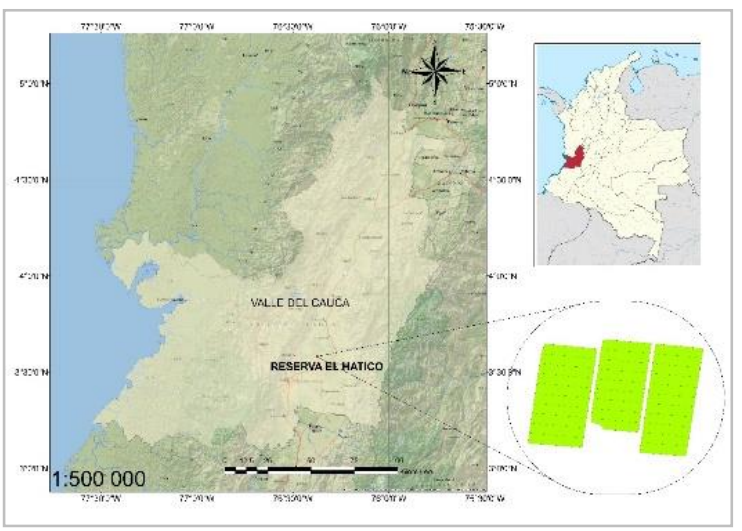

Figure 1. Location of experimental crop (758B)

\subsection{Spectroradiometer EPP2000}

The reflectance measured in each of the sampled plants was captured with a portable equipment that performs spectral measurements between $200 \mathrm{~nm}$ and $1100 \mathrm{~nm}$. This range covers the ultraviolet, visible and near infrared spectrum. The plants reflectance is measured with a spectral resolution of $0.5 \mathrm{~nm}$. The equipment was coupled with the fiber optic F400 - VISNIR of StellarNet Inc, this probe has an aperture of $400 \mathrm{um}$ and a field of view of (FOV) of $25.4^{\circ}$. The spectrometer used is shown in Figure 2. (StellarNet Inc, 2014).

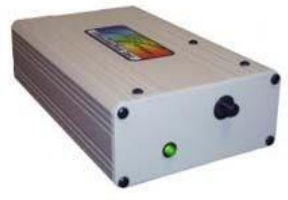

Figure 2. Spectroradiometer EPP2000.

\section{METHODOLOGY}

\subsection{Sampling and processing of hyperspectral data}

The spectral signatures used in this project were obtained from the reflectance measured with the EPP2000 spectrometer (StellarNet) between 200 to $1100 \mathrm{~nm}$. The optical fiber coupled to the equipment has a vision field (FOV) of $25.4^{\circ}$, which captured an area of $2 \mathrm{~cm}$ of radius at a distance of approximately $8 \mathrm{~cm}$. The data were collected in July 10th, 2018, in the 10th month of the sugarcane phenological cycle. The campaign was held between 10 a.m. and 3 p.m. for the purpose of maintaining an angle of $0-30^{\circ}$ between the sun and the zenith.

To avoid the edge effect, the samples were taken $20 \mathrm{~m}$ after the border edge of the two central grooves (Figure 3). At this point, 10 plants that didn't show spots, diseases or lesions at the foliar level were chosen out of the rest. Additionally, those plants were properly developed taking into account their growth phase. From each plant a spectral signature was obtained by pointing the optical fiber on the TVD leaf.

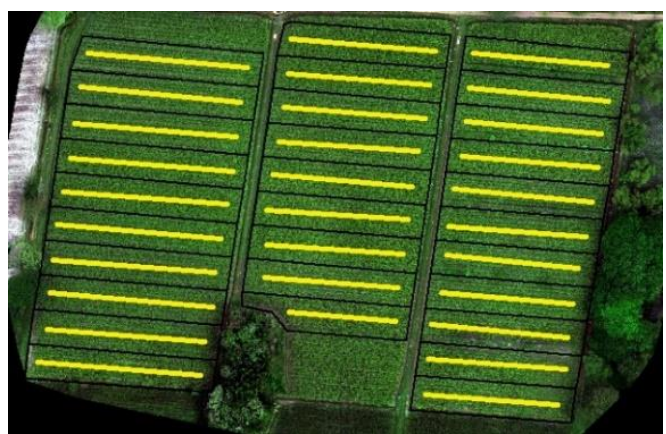

Figure 3. Selection of central grooves in each plot

The spectral signature corresponds to an average of 5 measurements of the same leaf. Before starting the evaluation, the minimum and maximum reflectance were calibrated with the spectralon to reduce samples variation (Suarez, Apan \& Werth, (2016).

A standardization process was carried out in order to introduce the spectral signatures into the neural network. The data were scaled according to the upper and lower limit of the data range with a variation from 0 to 1 .

\subsection{Artificial neural networks}

\section{Autoencoder}

Autoencoder is a type of neural network that uses the same sequential model used by the MLP, however, its design changes due to the low quantity of neurons found in the hidden layers in comparison to the output layers. The autoencoder is based on the dimensionality reduction obtained from the analysis of main components (PCA), which take the greatest weight 
characteristics in the input data and reduce them through linear transformations.

Although the autoencoder and the PCA are similar, the autoencoder uses a nonlinear component analysis due to high data variation. As shown in Figure 4, the autoencoder is composed of two regions: the encoding layer and the decoding layer. The encoding layer is where the data is transformed reducing its dimensionality and then transporting the important information of the group data to the hidden layers. On the other hand, the decoding layer, is where the network rebuilds the data following the scheme of the input layer.

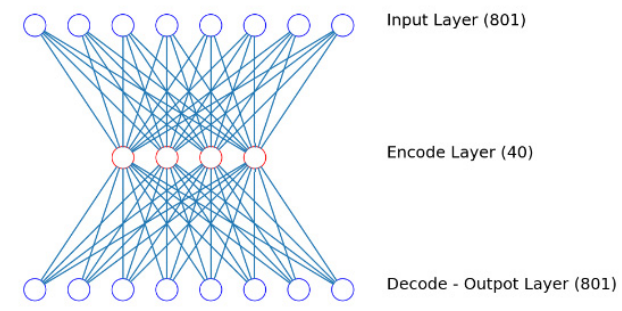

Figure 4. Autoencoder Architecture

During the process, the 300 sampled spectral signatures (each with 801 wavelengths) were organized in a $300 \times 801$ matrix. $80 \%$ of the data set is used for network training and the other $20 \%$ is used for prediction and validation of the productivity estimation model. The matrices of $240 \times 801$ and $60 \times 801$ entered to an autoencoder with an input layer of 801 neurons and then to a hidden or encoded layer with 40 neurons. The layer encoded with 40 neurons within the autoencoder architecture results in two matrices: $240 \mathrm{x} 40$ and $60 \mathrm{x}$ 40. Finally, the network has an output (or decoded layer) with 801 neurons. The hidden layer has an exponential linear unit (ELU) activation function (Eq. 1), which is a variation of the rectified linear unit (ReLU) function.

$$
f(x)=\left\{\begin{array}{c}
\alpha\left(e^{x}-1\right) \text { para } x<0 \\
x \text { para } x>0
\end{array}\right.
$$

Where: $\mathrm{e}=$ exponential constant

$$
\mathrm{x}=\text { input value }
$$

The main difference between the two activation functions is the input values. When they are close to 0 or negative, ReLU has a gradient that turns them into 0 and the network can't propagate the error backwards, it means the neural artificial network can't be trained with the input data. On the other hand, the ELU function provides a slope in the negative quadrant of the function, then neurons are activated with these values and obtaining a more accurate result for problems with this type of data.
The Sigmoidal activation function (Eq. 2) was implemented in the hidden layer, this allows a constant learning rate, avoiding slow rhythms where the network can remain stuck in a local minimum; also avoids high rhythms where instability in the error function is generated, with jumps in the weights close to the minimum that don't allow to reach it.

$$
f(x)=\frac{1}{1-e^{-x}}
$$

\section{Multilayer Perceptron}

The MLP is a widely used neural network. It is based on a back propagation algorithm, which distributes the error of the output layer, in the hidden layers. This network architecture is made up of an input layer, at least one hidden layer and one output layer. Connections between these types of neural network are generally either FeedForWard or sequential, which means that the input layer neurons are connected to the neurons in the hidden layer, as shown in Figure 5. The mathematical concept of this class of neural network is presented in equation 2 (NG, Andrew, 2014)

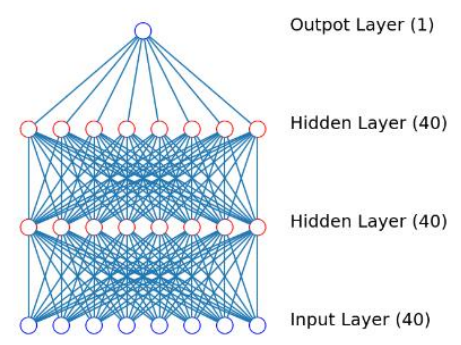

Figure 5. Architecture Perceptron Multilayer

Autoencoder showed two matrices: 240 x 40 and $60 \times 40$, which store the compression of the 801 wavelengths. The productivity vectors, obtained in the 2016, 2017 and 2018 harvests, are added to the new matrix before being incorporated into the MLP; obtaining matrices of $240 \times 41,240 \times 42,240 \times 43$ and $60 \times 41,60 \times 42,60 \times 43$ respectively. The architecture of the neural network is composed of an input layer containing 41 neurons, 2 hidden layers with 41 neurons each and finally, an output layer with a single neuron, which returns the productivity estimation within vector $60 \times 1$. The first hidden layer contains a hyperbolic tangent activation function; the second layer has an ELU function and the output layer was assigned a sigmoidal activation function.

\section{RESULTS}

The spectral signature shown in Figure 6 is the average result of the hyperspectral signatures obtained during the data acquisition campaign. This hyperspectral signature represents the spectral response's behaviour of the plant 
in different regions of the electromagnetic spectrum. During the process of photosynthesis, the chlorophyll pigments absorbed blue and red light, showing absorption peaks at 490 and $660 \mathrm{~nm}$, respectively (Araque and Jimenez 2009). This behavior is presented graphically at the minimum of the curve, where the reflectance has lower percentages. The green region is made up of bands where one of the maximum reflectance sites is generated, this represents the foliar surfaces of vegetation at $550 \mathrm{~nm}$. This maximum is caused by the low absorption of radiant energy, which produces a green pigmentation in plants.

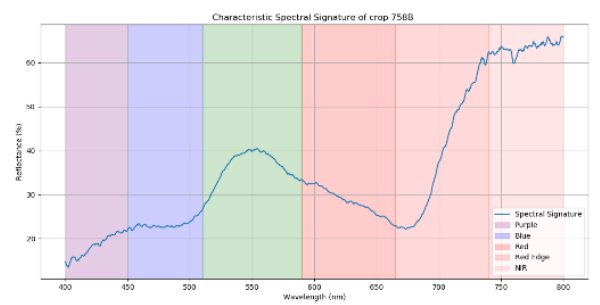

Figure 6. Average spectral signature of crop 758B

As seen in Figure 6, the highest reflectance values of the hyperspectral signature are at the near infrared region (NIR), due to the vegetation's health reflects the reflectance found in the wavelength. This region is commonly used to classify vegetation and identify stress on crops. Finally, the Red-Edge band, which is in the middle of infrared and red spectrum, shows a high inclination where absorption levels decrease as it moves towards larger wavelengths.

To estimate the productivity of sugarcane, two neural networks were implemented. At the beginning, the data is added to the Auto-encoder through the matrices of $240 \times 801$ and $60 \times 801$, where $95 \%$ of compression of the data is obtained using the synaptic weights calculated in the encoding phase, reducing the matrices to 40 columns. The learning process of the neural network is presented in Figure 7. The overall fit of the learning model had a mean square error (MSE) value below 0.02 for 50 iterations. This value reduces as the iterations increase, finding an accuracy close to 0 for 400 iterations.

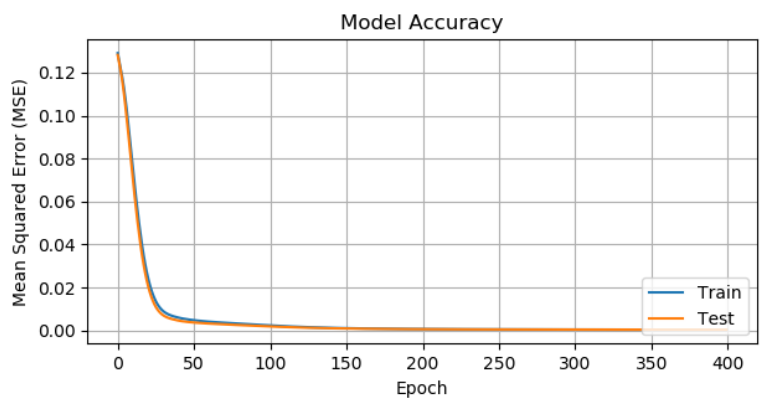

Figure 7. Autoencoder precision model
The productivity vectors of the previous crops were added to the new matrices to be included in the MLP. The new training matrix is $240 \times 43$, where columns 41,42 and 43 contain the productivity delivered by Cenicaña in 2016, 2017 and 2018 harvest, respectively. $20 \%$ of the remaining data is divided into a $60 \times 42$ matrix that contains in columns 41 and 42 the productivity of 2016 and 2017 harvests respectively, this matrix is used to make the 2018 productivity prediction. The other 60 productivity data, stored in a vector, is used to validate the model with the predicted productivity.

In this new neural network, data was used to estimate the biomass in the crop. The training of the MLP is shown in Figure 8 . The learning process is presented in a slower way than in the autoencoder, where the MSE values below 0.02 were given only until 300 iterations, the graphic also shows a noise due to the dimensionality and the amount of training data.

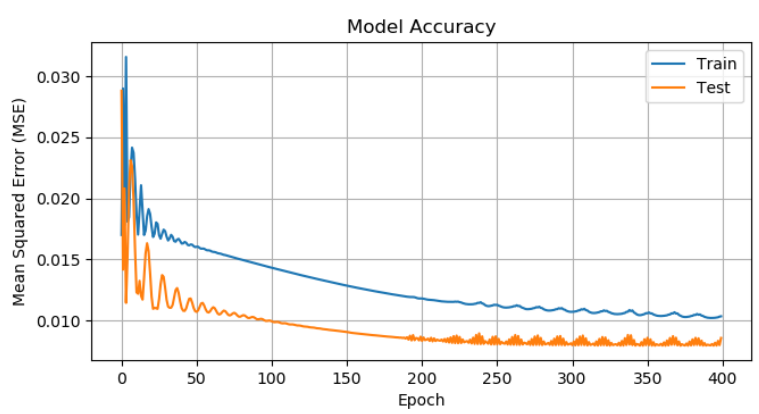

Figure 8. MLP precision model

The low MSE values in the learning models of the neural network (presented in the graphs) refer to the backpropagation algorithm in which the architectures are based. In the supervised learning process, this algorithm modifies the synaptic weights in its layers to obtain the closest result to the output data provided.

The prediction of the productivity model is developed by including in the neural network the matrix $60 \times 42$, that has the compressed reflectance and the productivity of the 2016 and 2017 harvest. The neural network returns a vector with 60 estimated productivity values that are compared with the real productivity values of each of the plots related to the compressed spectral signatures. The evaluation of the final estimation model has an absolute mean error (AME) of $8.06 \mathrm{t} /$ ha and an RMSE of 10.43 $\mathrm{t} / \mathrm{ha}$, giving an accuracy of $91.08 \%$. By adding only the 2018 harvest productivity, the estimate had an accuracy of $85.37 \%$ and $88.47 \%$ with the productivity of 2017 and 2018 when considered in training. 
Linear regression Estimated PPN vs. Real PPN

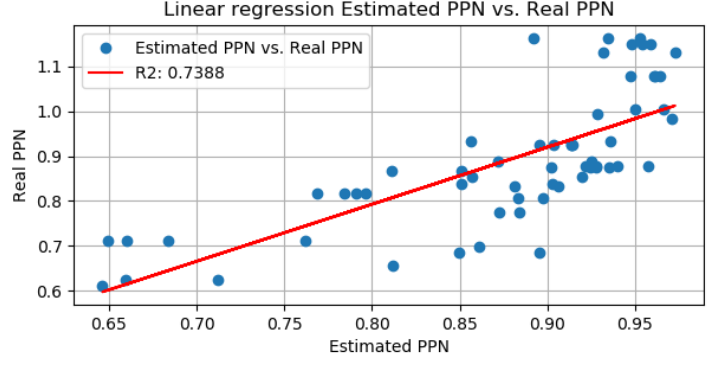

Figure 8. Lineal regression between Estimated PPN and Real PPN.

Figure 8 shows the linear regression obtained between the estimated productivity with the MLP and the actual data productivity provided by Cenicaña. The regression obtained a coefficient of determination R2 of 0.7388 , which indicates a low dispersion of the data, in which the error is distributed evenly in the plots. The linear regression has a correlation directly proportional to the precision values obtained in the estimate. The productivity estimation is globally calculated on the crop without discriminating the different nitrogen fertilization treatments, so the linear regression doesn't allow a better adjustment.

\section{CONCLUSIONS}

In this project, two types of artificial neural network were evaluated with the purpose of estimating sugarcane productivity. Initially, the autoencoder achieved a correct compression of the reflectance data to $95 \%$, reducing the dimensionality of the input matrix to only 40 columns that keep the spectral crop information. A compression greater than $95 \%$, generated a higher loss of collected data; in addition, it increased noise in the autoencoder learning process returning matrix's columns without values.

The normalization of the input data is a process that standardizes the scale of variables that enter into the input layer of the neural network and reduces the computation workload. Thus, the training process is more efficient and the results are more accurate. This procedure avoided biases that could occur due to outliers corresponding to the scalar variation of the inputs.

The autoencoder was a solution for the problem of dimensionality caused in an MLP when entering a matrix with a greater number of variables from the collected samples. The $300 \times 40$ matrix and the productivity vectors used to train the MLP, enabled a productivity estimation with an accuracy between 85 to $92 \%$. The inclusion of productivity data from previous crops made possible an improving for the performed estimations. Also, a prediction accuracy of $85.37 \%$ was obtained using only the 2018 harvest. Besides that, the prediction accuracy was increased to $88.47 \%$, including the productivity of 2017 . Finally, when the 3 productivity vectors of 2016 , 2017 and 2018 harvest were included, the accuracy of the prediction increased to $91.23 \%$ due to the consideration of productivity variation in each year. This variation indicates that productivity behavior is a phenomenon that doesn't follow a sequential pattern over time even under experimental conditions, therefore, the use of a greater amount of information from previous periods, brings more precise behavior modelling trends to obtain more accurate results.

\section{REFERENCES}

Abdel-Rahman, E. M., van den Berg, M., Way, M. J., \& Ahmed, F. B. (2009, July). Hand-held spectrometry for estimating thrips (Fulmekiola serrata) incidence in sugarcane. In 2009 IEEE International Geoscience and Remote Sensing Symposium (Vol. 4, pp. IV-268). IEEE.

Araque, L., \& Jiménez A, 2009. Caracterización de firma spectral a partir de sensors remotos para el manejo de sanidad vegetal en el cultivo de Palma de Aceite. Revista Palmas, 30(3), 63-79.

Asocaña, 2018. Aspectos Generales del Sector Agroindustrial de la Caña 2017-2018.

Bégué, A., Lebourgeois, V., Bappel, E., Todoroff, P., Pellegrino, A., Baillarin, F., \& Siegmund, B. (2010). Spatio-temporal variability of sugarcane fields and recommendations for yield forecast using NDVI. International Journal of Remote Sensing, 31(20), 53915407.

Caicedo, F., López, J., 2009. A practical approach to artificial neural networks, Editorial Universidad del Valle, Santiago de Cali.

Curran, P. J., Dungan, J. L., \& Peterson, D. L. (2001). Estimating the foliar biochemical concentration of leaves with reflectance spectrometry: testing the Kokaly and Clark methodologies. Remote Sensing of Environment, 76(3), 349-359.

Duveiller, G., López - Lozano, R., \& Baruth B, 2013, Enchanced Processing Of $1-\mathrm{Km}$ spatial resolution fAPAR time series for sugarcane Yield Forescasting And monitoring. Remote Sensing, 5(3), 1091-1116.

Espín, T., 2015, Comparación de métodos de clasificación de imágenes de satélite en la cuenta del Río Argos (Región de Murcia), 327-348. 
NG, A. (2011). Sparse autoencoder. CS294A Lecture notes, 72(2011), 1-19.

Suarez, L. A., Apan, A., \& Werth, J. (2016). Hyperspectral sensing to detect the impact of herbicide drift on cotton growth and yield. ISPRS Journal of Photogrammetry and Remote Sensing, 120, 65-76.

StellarNet, 2014, Miniaiture Spectrometer Manual. 